\title{
Syndrome of inappropriate antidiuretic hormone secretion in patients with brucellosis
}

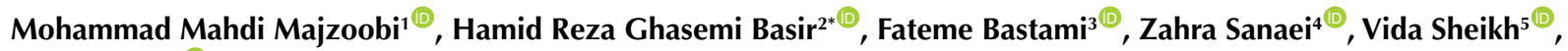 \\ Peyman Eini ${ }^{6}$
}

'Brucellosis Research Center, Hamadan University of Medical Sciences, Hamadan, Iran

${ }^{2}$ Department of Pathology, School of medicine, Hamadan University of Medical Sciences, Hamadan, Iran

${ }^{3}$ School of medicine, Hamadan University of Medical Sciences, Hamadan, Iran

${ }^{4}$ Department of Community medicine, School of medicine, Hamadan University of Medical Sciences, Hamadan, Iran

5Department of Nephrology, School of medicine, Hamadan University of Medical Sciences, Hamadan, Iran

${ }^{6}$ Department of infectious disease, School of Medicine, Hamadan University of Medical Sciences, Hamadan, Iran

\section{*Correspondence to Hamid Reza Ghasemi Basir, Email: hrgb2004@yahoo.com ha.ghasemi@umsha.ac.ir}

Received 11 Apr. 2021 Accepted 10 June 2021 Published online 28 June 2021

Keywords: SIADH, Brucellosis

Abstract
Introduction: Syndrome of inappropriate antidiuretic hormone secretion (SIADH), is generally characterized by
hyponatremia and hypo-osmolarity.
Objectives: SIADH may develop following some infectious diseases including brucellosis, which regarding the
severity of the disease may cause morbidity or mortality.
Patients and Methods: In this case-control study, 110 patients with brucellosis and 110 healthy controls were
enrolled. The case group was selected among patients having clinical signs and symptoms of brucellosis with a
Wright test titer $\geq 1 / 160$ and a $2 \mathrm{ME}$ test titer $\geq 1 / 80$. About 10 cc blood sample was taken from each of them to test
the blood sugar, thyroid stimulating hormone (TSH), serum sodium, serum potassium, blood urea nitrogen (BUN),
creatinine, and serum cortisol. Random urine samples were also taken from participants to measure the urinary
Osmolarity and sodium.
Results: The frequency of hyponatremia (Na $<135$ mEq/L) in patients with brucellosis was significantly higher
than the control group but there was no significant difference between the groups in the frequency of abnormal
urinary sodium (more than $40 \mathrm{mEq} / \mathrm{L}$ ). Meanwhile, the frequency of abnormal uric acid (less than 4 mg/dL)
was significantly higher in patients than the control group. The frequency of abnormal urine osmolarity (above
300 mOsm/kg) was significantly higher in patients than the control group. In total, only 9 out of 110 patients
had SIADH with asymptomatic mild hyponatremia and severe hyponatremia was seen only in one patient with
prolonged Brucella meningitis.
Conclusion: Based on the defined criteria, $8.2 \%$ of brucellosis patients were at the risk of SIADH, but the findings
were in favor of asymptomatic SIADH without prominent hyponatremia.

Introduction

Brucellosis is the most common zoonosis caused by gram-negative coccobacillus called "Brucella spp", which its global human incidence is more than 500000 cases, annually. Although it was identified more than a century ago, brucellosis is still considered as one of the most important common diseases among humans and animals in many countries, particularly in the Mediterranean and the Middle East region. Animal and human brucellosis are highly prevalent and endemic in Iran so that the country is ranked as the fourth concerning of the global incidence of brucellosis (1).

Humans may be infected through the consumption of unpasteurized milk and dairy products, contaminated meat, or direct contact with the secretions of infected

\begin{abstract}
Key point
If hyponatremia is observed in patients with Brucella, inappropriate antidiuretic hormone secretion should be considered to prevent complications.
\end{abstract}

animals. Its clinical presentations include a wide range, from asymptomatic infection to acute, subacute, and chronic forms. The main symptoms of brucellosis include arthralgia, fever, sweating, weight loss, and musculoskeletal pain (2).

It may occur with a variety of clinical syndromes, including musculoskeletal, typhoidal brucellosis, neuroborreliosis, and epididymo-orchitis. Different degrees of syndrome of inappropriate antidiuretic hormone secretion (SIADH), which is generally characterized by hyponatremia and

\footnotetext{
Copyright $(\odot 2022$ The Author(s); Published by Nickan Research Institute. This is an open-access article distributed under the terms of the Creative Commons Attribution License (http://creativecommons.org/licenses/by/4.0), which permits unrestricted use, distribution, and reproduction in any medium, provided the original work is properly cited.
} 
hypo-osmolarity, may develop following some infectious diseases, including brucellosis, which regarding the severity of the disease may cause morbidity or mortality. Common manifestations of this syndrome include general weakness and attention disorder in mild to moderate cases, and encephalopathy and brain edema in severe type (3).

Hyponatremia (serum sodium level less than 135 $\mathrm{mEq} / \mathrm{L}$ ) is the most common electrolyte disorder in hospitalized patients, which one of its main and dangerous complications is brain edema and encephalopathy (4). This electrolyte disorder may be caused by increased sodium excretion or decreased free fluid excretion, which can be determined by measuring urine osmolarity. A urine sodium concentration less than $25 \mathrm{mEq} / \mathrm{L}$ reveals the effective volume loss, while urinary sodium concentration above $25 \mathrm{mEq} / \mathrm{L}$ indicates inappropriate renal function, consumption of diuretics or SIADH. Antidiuretic hormone $(\mathrm{ADH})$ is released from the posterior portion of pituitary gland in response to an increase of plasma osmolarity, which in turn causes reabsorption of water in the kidneys and its concentration returns to the baseline secondary to dilution of plasma. Occasionally, in some patients, this hormone continues to be released despite normal or low plasma osmolarity, leading to euvolemic hyponatremia, disproportionate increase in urinary osmolarity and a decrease in serum osmolarity, known as $\mathrm{SIADH}(5,6)$. Central nervous system disorders, some tumors, lung diseases including pulmonary tuberculosis, endocrine disorders, paraneoplastic syndromes, and some medications such as anticonvulsants, anti-Parkinson, angiotensin-converting enzyme inhibitors, may cause SIADH (3,7-10).

The best laboratory method to diagnose this complication is simultaneous measurement of blood and urine vasopressin as well as sodium and osmolarity of plasma and urine; however, there is an easier method which uses eight indicators: Hypotonic hyponatremia, high urinary osmolarity (more than what expected for this hyponatremia), and a urinary sodium more than 20 $\mathrm{mEq} / \mathrm{L}$, along with normal renal function, normal function of endocrine glands (thyroid, pituitary and adrenal), absence of hypovolemia, lack of edema, and response to the fluid restriction (11).

Since untreated hyponatremia in infectious diseases such as brucellosis may lead to severe complications including confusion, delirium, muscle weakness, hyporeflexia, seizures, and eventually cerebral edema, encephalopathy, and coma (12-14), identification and treatment of hyponatremia in patients with brucellosis helps to prevent these complications and determining its frequency in brucellosis endemic areas can be useful for physicians to pay special attention to this complication.

\section{Objectives}

SIADH may develop following infectious diseases including brucellosis, which regarding the severity of the disease may cause morbidity or mortality. In this current study, patients with brucellosis are investigated to identify the frequency of SIADH.

\section{Patients and Methods}

Study design

In this prospective descriptive-analytical study, 220 subjects including 110 brucellosis patients (aged more than 15 years) and 110 healthy controls who were referring to Sina hospital of Hamadan city (west of Iran), are studied from 2018 to 2019. Participants were selected using the convenience sampling method among patients having clinical signs of brucellosis along with a Wright titer equal or more than $1 / 160$ and a $2 \mathrm{ME}$ titer equal or more than $1 / 80$ (3). After obtaining written informed consent from all participants, $10 \mathrm{cc}$ blood sample was taken for testing blood glucose, thyroid stimulating hormone (TSH), serum sodium, serum potassium, blood urea nitrogen (BUN), creatinine, and serum cortisol. Random urine samples were also taken from all participants to measure the urinary sodium and osmolarity. All patients and participants in the control group were evaluated for the frequency of SIADH diagnostic criteria $(3,6)$ including euvolemic hyponatremia (serum sodium less than $135 \mathrm{mEq} / \mathrm{L}$ ), serum hypo-osmolarity (serum osmolarity less than 275 $\mathrm{mOsm} / \mathrm{L}$ ), increased urinary sodium to more than 40 $\mathrm{mEq} / \mathrm{L}$, decreased serum uric acid to less than $4 \mathrm{mg} / \mathrm{dL}$, urinary osmolarity above $300 \mathrm{mOsm} / \mathrm{kg}$, absence of renal, thyroid and adrenal disorders, and absence of antidiuretics consumption. Serum osmolarity was calculated according to following formula: Serum Osmolarity $=2 \times($ Serum $\mathrm{Na}$ + Serum K) + Glucose/18 + BUN/2.8

Exclusion criteria were; liver failure, heart failure, renal failure, patients with brain edema or brain (pituitary) tumor, thyroid disorders, adrenal disorders, CNS infections, pneumonia, pulmonary tuberculosis, consumption of diuretics and antidiuretics, anticonvulsants, anti-Parkinson drugs, angiotensin-converting enzyme inhibitors, and patients with a history of previous brucellosis.

\section{Data analysis}

Data were analyzed using SPSS version 16 (SPSS Inc., Chicago, IL, USA). Descriptive information extracted from qualitative data was described in the form of tables, graphs, ratios, and percentages. To summarize quantitative variables, central and dispersion index were used. The normality of data was examined using the Kolmogorov-Smirnov test. Among the variables, age, uric acid, and urinary sodium had a normal distribution $(P>0.05)$, and the rest were slightly abnormal $(P<0.05)$. The chi-square test was used to determine the frequency of SIADH for qualitative, nominal, and rank variables. Besides, to determine the frequency of SIADH based on the quantitative variables, the student $t$ test, and MannWhitney $U$ test were used. All analyzes were performed at a $95 \%$ confidence level, and the significance level was 
considered less than 0.05 .

The sample size was calculated to 86 subjects in each group, based on the study conducted by Golsahani et al (1) with 95\% confidence level and 95\% statistical power, but to increase the study power, 110 subjects were enrolled in each group.

\section{Results}

In this descriptive-analytical study which aimed to determine the frequency of SIADH in brucellosis and to compare the results with a control group, 220 subjects (110 patients with brucellosis and 110 healthy controls) were studied. Frequency of males and females was $66.4 \%$ and $33.6 \%$ in brucellosis patients, and $48.2 \%$ and $51.8 \%$ in the control group, respectively $(P=0.006)$. The mean ( \pm standard deviation) age of patients with brucellosis and control group were $43.97( \pm 16.30)$ and $44.68( \pm 17.09)$ years, respectively $(P=0.328)$. In terms of location, $22.7 \%$ and $77.3 \%$ of patients with brucellosis and $73.6 \%$ and $26.4 \%$ of the control group were urban and rural, respectively $(P=0.001)$. Occupationally, 71 patients (64.5\%) were exposed to brucellosis with working in a butchery, slaughterhouse and animal husbandry. In addition, 101 patients (91.8\%) had a history of consuming local dairy products.

Among clinical syndromes, 102 (92.7\%) subjects had musculoskeletal involvement, 3 (2.7\%) had orchitis, and $5(4.5 \%)$ had typhoidal syndrome. One case of brucella meningitis was also hospitalized, which was excluded due to central nervous system disease.

As shown in Table 1, and according to the results of the chi-square test, the frequency of hyponatremia $(\mathrm{Na}<135$ $\mathrm{mEq} / \mathrm{L})$ in patients with brucellosis was significantly higher than the control group ( $8.2 \%$ versus $1.8 \%)(P=0.030)$.

The prevalence of abnormal urinary sodium status (more than $40 \mathrm{mEq} / \mathrm{L}$ ) in 80 patients whose urinary sodium levels were tested and in the control group was $96.2 \%$ and $97.3 \%$, respectively (Table 1 ). According to the chi-square test result, no significant difference was found between them $(P=0.691)$.

Additionally, the frequency of abnormal uric acid (less than $4 \mathrm{mg} / \mathrm{dL}$ ) in 86 patients whose uric acid levels were measured and in the control group was $32.56 \%$ and $16.4 \%$, respectively (Table 1 ). According to the chisquare test result, the frequency of abnormal uric acid was significantly lower for patients than the control group $(P$ $=0.008)$.

The frequency of abnormal urine osmolarity (above 300 $\mathrm{mOsm} / \mathrm{kg}$ ) in patients and the control group were $86.4 \%$ and $70.9 \%$, respectively (Table 1 ). According to the chisquare test, the frequency of abnormal urine osmolarity in patients was significantly higher than the control group $(P$ $=0.005)$. In addition, serum osmolarity less than 275 was not observed in any of the patients and the control group.

Abnormal serum cortisol (less than $5 \mathrm{ng} / \mathrm{mL}$ ) was not seen in any of the participants, which indicates the absence of adrenal insufficiency in both groups. Furthermore, abnormal TSH (in favor of hypo or hyperthyroidism) was not identified in any of the participants, which indicates the absence of significant thyroid disorders among patients and healthy controls.

Moreover, there was no statistically significant difference between patients and the control group in terms of the frequency of abnormal urea $(>20 \mathrm{mg} / \mathrm{dL})$ and abnormal creatinine $(>1.5 \mathrm{mg} / \mathrm{dL} ; P=0.068$ and $P=0.230)$, which indicate the absence of significant renal failure in the patient group.

Although $9(8.2 \%)$ out of 110 brucella patients had SIADH by defined criteria, their serum sodium levels decreased slightly (133-134 mEq/L) and therefore appear to be mainly mild asymptomatic hyponatremia. Severe hyponatremia of $119 \mathrm{mEq} / \mathrm{L}$ was reported in only one patient with prolonged brucella meningitis.

According to the findings of Table 2, the mean of urinary sodium and urine osmolarity in brucellosis patients were significantly higher than the control group, and the mean of serum cortisol and uric acid in patients were significantly lower than the control group. However, there was no statistically significant difference between the mean of serum sodium level, serum osmolarity and BUN level between the two groups. Sum of these findings are in favor of increasing the risk of asymptomatic SIADH (without obvious hyponatremia) in brucellosis patients compared to the control group.

Table 1. Comparison of frequency of SIADH diagnostic criteria between Brusellosis and healthy control groups

\begin{tabular}{|c|c|c|c|c|}
\hline Criteria of SIADH & & $\begin{array}{c}\text { Case (Brusellosis) } \\
\text { No. }(\%)\end{array}$ & $\begin{array}{l}\text { Healthy control } \\
\text { No. }(\%)\end{array}$ & $P$ value ${ }^{a}$ \\
\hline \multirow{2}{*}{ Serum $\mathrm{Na},<135 \mathrm{mEq} / \mathrm{L}$} & Yes & $9(8.2)$ & $2(1.8)$ & \multirow{2}{*}{0.030} \\
\hline & No & $101(91.8)$ & $108(98.2)$ & \\
\hline \multirow{2}{*}{ Urine $\mathrm{Na},>40 \mathrm{mEq} / \mathrm{L}$} & Yes & $77(96.2)$ & $107(97.3)$ & \multirow{2}{*}{0.691} \\
\hline & No & $3(3.8)$ & $3(2.7)$ & \\
\hline \multirow{2}{*}{ Serum uric acid, $<4 \mathrm{mg} / \mathrm{dL}$} & Yes & $28(32.56)$ & $18(16.4)$ & \multirow{2}{*}{0.008} \\
\hline & No & $58(67.44)$ & $92(83.3)$ & \\
\hline \multirow{2}{*}{ Urine osmolarity, >300 mOsm $/ \mathrm{kg}$} & Yes & $95(86.4)$ & $78(70.9)$ & \multirow{2}{*}{0.005} \\
\hline & No & $15(13.6)$ & $32(29.1)$ & \\
\hline \multirow{2}{*}{ Serum osmolarity, $<275 \mathrm{mOsm} / \mathrm{L}$} & Yes & $0(0)$ & $0(0)$ & \multirow{2}{*}{1} \\
\hline & No & $110(100)$ & $110(100)$ & \\
\hline
\end{tabular}

${ }^{a}$ Chi-square test. 
Table 2. Comparisone of SIADH related biomarkers (Mean \pm SD) between case and control groups

\begin{tabular}{|c|c|c|c|}
\hline \multirow{2}{*}{ Variable } & \multicolumn{2}{|c|}{ Group } & \multirow{2}{*}{$P$ value } \\
\hline & Case (Brusellosis) & Healthy control & \\
\hline Serum $\mathrm{Na}($ Mean $\pm \mathrm{SD}), \mathrm{mEq} / \mathrm{L}$ & $140.46(4.07)$ & $140.04( \pm 1.88)$ & $0.319^{\mathrm{a}}$ \\
\hline Urine $\mathrm{Na}($ Mean $\pm \mathrm{SD}), \mathrm{mEq} / \mathrm{L}$ & $143.39( \pm 60.8)$ & $123.66( \pm 56.62)$ & $0.023 b$ \\
\hline Serum uric acid (Mean \pm SD), mg/dL & $4.68( \pm 0.15)$ & $5.36( \pm 0.13)$ & $0.001 b$ \\
\hline Urine osmolarity (Mean $\pm \mathrm{SD}$ ), mOsm/kg & $574.09( \pm 195.79)$ & $510.82( \pm 219.94)$ & $0.025^{\mathrm{a}}$ \\
\hline Serum osmolarity (Mean $\pm \mathrm{SD}$ ), mOsm/L & $300.65( \pm 8.90)$ & $299.62( \pm 4.73)$ & $0.282^{\mathrm{a}}$ \\
\hline
\end{tabular}

a Mann-Whitney $U$, b Student $t$ test.

Among the diagnostic criteria of SIADH, the frequency of hypouricemia was significantly higher in women (50\%) than men $(23.2 \%)$ and the frequency of urinary osmolarity above $300 \mathrm{mOsm} / \mathrm{kg}$ in men $(80.8 \%)$ was higher than women $(51.4 \%) \quad(P=0.001$ and $P=0.012)$. Also, the frequency of hyponatremia, urinary sodium more than 40 $\mathrm{mEq} / \mathrm{L}$ and serum osmolarity less than $275 \mathrm{mOsm} / \mathrm{L}$ were not significantly different between the men and women with brucellosis $(P>0.05)$.

The mean age of patients with euvolemic hyponatremia (42.61 \pm 15.7 years) was significantly lower than patients without hyponatremia $(59.67 \pm 14.33$ years $)$ based on the non-parametric Mann-Whitney $U$ test $(P=0.014)$. However, there was no statistically significant difference between the mean age of patients with hypouricemia, urinary osmolarity above $300 \mathrm{mOsm} / \mathrm{kg}$, urinary sodium more than $40 \mathrm{mEq} / \mathrm{L}$ and serum osmolarity less than 275 $\mathrm{mOsm} / \mathrm{L}$ and patients without these criteria $(P>0.05)$.

Also, there was no statistically significant difference between the mean duration of brucellosis in patients with hyponatremia, hypouricemia, urinary osmolarity above $300 \mathrm{mOsm} / \mathrm{kg}$, urinary sodium more than $40 \mathrm{mEq} / \mathrm{L}$ and serum osmolarity less than $275 \mathrm{mOsm} / \mathrm{L}$ and patients without them $(P>0.05)$, according to the results of MannWhitney U test.

\section{Discussion}

In the current study, 110 patients with brucellosis were compared with 110 healthy subjects concerning the incidence of SIADH according to the laboratory findings. Overall, the results indicated a higher incidence of this syndrome among patients. Based on the diagnostic criteria of SIADH, in the brucellosis group, 9 subjects (8.2\%) had hyponatremia, 77 patients $(96.2 \%)$ had urinary sodium more than $40 \mathrm{mEq} / \mathrm{L}, 28$ participants (32.6\%) had hypouricemia, and 95 patients $(86.4 \%)$ had urinary osmolarity above $300 \mathrm{mOsm} / \mathrm{kg}$. Based on the aforementioned criteria, only 9 patients $(8.2 \%)$ had typical SIADH.

Other studies that investigated the coincidence of the SIADH including the following; the study conducted by Tagarro et al (15) on hospitalized children with communityacquired pneumonia, reported that hyponatremia is common but true SIADH is a rare event; Callewart et al (16) study on patients who had spinal surgery, reported a coincidence of $6.9 \%$; the study performed by Cui et al (17) reported $38.7-73 \%$ coincidence of hyponatremia in tuberculous meningitis and stated SIADH as the main cause in children; Moro et al (18), reported $16.8 \%$ incidence of hyponatremia during traumatic brain injury.

Dugler et al (19) investigated 35 patients with acute brucellosis in Turkey which 19 (54\%) of them had SIADH. Bala et al (3) investigated 160 patients with brucellosis, that 35 (21.9\%) of them had SIADH. In the current study, $8.2 \%$ of patients had typical SIADH with full diagnostic criteria. Differences observed in the results of the studies may be due to the differences in the disease severity or stage. Since brucellosis is endemic in Iran, despite having clinical symptoms and positive serologic results consistent with brucellosis, most of the referring patients are not in the acute or severe stage of disease to lead to symptomatic SIADH. Although some diagnostic criteria for SIADH, including urinary sodium above $40 \mathrm{mEq} / \mathrm{L}$ and urinary osmolarity above $300 \mathrm{mOsm} / \mathrm{kg}$, were observed in a high percentage of patients.

According to the diagnostic criteria, in the study conducted by Aysha et al (20), about 24\% of patients with brucellosis had low plasma osmolarity. Besides, they reported that in patients with brucellosis, SIADH occurs as asymptomatic hyponatremia, which is consistent with the results of the present study.

In a study by Dugler et al (19) on 35 patients with acute brucellosis, $57 \%$ of subjects had hypouricemia, and all of the patients had an increased level of urinary sodium. In the current study, $32.6 \%$ of patients had hypouricemia, and in $96.2 \%$ of cases, urinary sodium was more than 40 $\mathrm{mEq} / \mathrm{L}$ which is consistent with the study of Dugler et al.

In the current study, among diagnostic criteria of SIADH, the frequency of hypouricemia was significantly higher in women than men, and the frequency of urinary osmolarity more than $300 \mathrm{mOsm} / \mathrm{kg}$ was significantly higher in men than women. Besides, the mean age of patients with hyponatremia was significantly lower than that of patients without hyponatremia. In the current study, there was no statistically significant difference between the mean duration of disease in patients with and without diagnostic criteria of SIADH. Likewise, Bala et al (3) reported that there was no statistically significant difference in the incidence of SIADH in terms of age and gender. 


\section{Conclusion}

Although $8.2 \%$ of brucellosis patients were diagnosed with SIADH based on the defined criteria, their serum sodium levels decreased slightly (133-134 mEq/L), and therefore it appears to be mostly asymptomatic mild hyponatremia. Severe hyponatremia was reported in only one patient with prolonged Brucella meningitis. However, if hyponatremia is observed in patients with Brucella, SIADH should be considered to prevent complications. It seems that screening of brucellosis patients for SIADH is not necessary except in patients with neurological manifestations.

\section{Limitations of the study}

The limitation of the study was the failure to refer some patients to perform the requested tests, which maximum participation was achieved by educating and justifying the patients about the importance and consequences of the disease and following up the patients with contact numbers.

\section{Authors' contribution}

MMM and MRGB were the principal investigators of the study. FB, ZS, VS and PE were included in preparing the concept and design. MMM and MRGB revisited the manuscript and critically evaluated the intellectual contents. All authors participated in preparing the final draft of the manuscript, revised the manuscript and critically evaluated the intellectual contents. All authors have read and approved the content of the manuscript and confirmed the accuracy or integrity of any part of the work.

\section{Conflicts of interest}

The authors declare that they have no competing interests.

\section{Ethical issues}

The research followed the tenets of the Declaration of Helsinki. The Ethics Committee of Hamadan University of Medical Sciences approved this study. The institutional ethical committee at Hamadan University of Medical Sciences approved all study protocols (IR. UMSHA.REC.139608). Accordingly, written informed consent was taken from all participants before any intervention. This study was extracted from M.D/MSc thesis of Fateme Bastami at this university (Thesis \#9604132397). Moreover, ethical issues (including plagiarism, data fabrication, double publication) have been completely observed by the authors.

Funding/Support

This project was carried out by a grant supported by Hamadan University of Medical Sciences.

\section{References}

1. Gompo TR, Shah R, Tiwari I, Gurung YB. Sero-epidemiology and associated risk factors of brucellosis among sheep and goat population in the south western Nepal: a comparative study. BMC Vet Res. 2021;17:132. doi: 10.1186/s12917-021-02835-8.

2. Zheng R, Xie S, Lu X, Sun L, Zhou Y, Zhang Y, Wang K. A systematic review and meta-analysis of epidemiology and clinical manifestations of human brucellosis in China. Biomed Res Int. 2018;2018:5712920. doi: 10.1155/2018/5712920.

3. Bala KA, Doğan M, Kaba S, Akbayram S, Aslan O, Kocaman S, Bayhan GI, Üstyol L, Demir N. The syndrome of inappropriate secretion of anti-diuretic hormone (SIADH) and brucellosis.
Med Sci Monit. 2016;22:3129-34. doi: 10.12659/msm.899977.

4. Fraser CL, Arieff Al. Epidemiology, pathophysiology, and management of hyponatremic encephalopathy. Am J Med. 1997;102:67-77. doi: 10.1016/s0002-9343(96)00274-4.

5. DiCarlo JV, Frankel LR. Neurologic stabilization. In: Behrman RE, Kliegman RM. Nelson Textbook of Pediatric. 17th ed. Philadelphia: Saunders; 2004. p. 311.

6. Oh JY, Shin JI. Syndrome of inappropriate antidiuretic hormone secretion and cerebral/renal salt wasting syndrome: similarities and differences. Front Pediatr. 2015;2:146. doi: 10.3389/ fped.2014.00146.

7. Srivatsa A, Majzoub JA: Disorders of water homeostasis. In: Lifshitz F, ed. Pediatric Endocrinology. New York: Informa Healthcare; 2007. p. 651-92.

8. Khushman M, Morris MI, Diaz L, Goodman M, Pereira D, Fuller K, et al. Syndrome of inappropriate anti-diuretic hormone secretion secondary to strongyloides stercoralis infection in an allogeneic stem cell transplant patient: a case report and literature review. Transplant Proc. 2017;49:373-377. doi: 10.1016/j.transproceed.2016.12.012.

9. Dhawan A, Narang A, Singhi S. Hyponatraemia and the inappropriate ADH syndrome in pneumonia. Ann Trop Paediatr. 1992;12:455-62. doi: 10.1080/02724936.1992.11747614.

10. Padilla G, Ervin MG, Ross MG, Leake RD. Vasopressin levels in infants during the course of aseptic and bacterial meningitis. Am J Dis Child. 1991;145:991-3. doi: 10.1001/ archpedi.1991.02160090043019.

11. Esposito P, Piotti G, Bianzina S, Malul Y, Dal Canton A. The syndrome of inappropriate antidiuresis: pathophysiology, clinical management and new therapeutic options. Nephron Clin Pract. 2011;119:c62-73. doi: 10.1159/000324653.

12. John CA, Day MW. Central neurogenic diabetes insipidus, syndrome of inappropriate secretion of antidiuretic hormone, and cerebral salt-wasting syndrome in traumatic brain injury. Crit Care Nurse. 2012;32:e1-7; quiz e8. doi: 10.4037/ccn2012904.

13. Kamoi K, Toyama M, Ishibashi M, Yamaji T. Hyponatremia and osmoregulation of vasopressin secretion in patients with intracranial bleeding. J Clin Endocrinol Metab. 1995;80:290611. doi: 10.1210/jcem.80.10.7559873.

14. Yee AH, Burns JD, Wijdicks EF. Cerebral salt wasting: pathophysiology, diagnosis, and treatment. Neurosurg Clin N Am. 2010;21:339-52. doi: 10.1016/j.nec.2009.10.011.

15. Tagarro A, Martín MD, Del-Amo N, Sanz-Rosa D, Rodríguez Md PhD M, Galán Md PhD JC, Otheo E. Hyponatremia in children with pneumonia rarely means SIADH. Paediatr Child Health. 2018;23:e126-e133. doi: 10.1093/pch/pxy003.

16. Callewart CC, Minchew JT, Kanim LE, Tsai YC, Salehmoghaddam S, Dawson EG, Delamarter RB. Hyponatremia and syndrome of inappropriate antidiuretic hormone secretion in adult spinal surgery. Spine (Phila Pa 1976). 1994;19:1674-9. doi: 10.1097/00007632-199408000-00004.

17. Cui H, He G, Yang S, Lv Y, Jiang Z, Gang X, Wang G Inappropriate antidiuretic hormone secretion and cerebral saltwasting syndromes in neurological patients. Front Neurosci. 2019;13:1170. doi: 10.3389/fnins.2019.01170.

18. Moro N, Katayama Y, Igarashi T, Mori T, Kawamata T, Kojima J. Hyponatremia in patients with traumatic brain injury: incidence, mechanism, and response to sodium supplementation or retention therapy with hydrocortisone. Surg Neurol. 2007;68:387-93. doi: 10.1016/j.surneu.2006.11.052.

19. Dulger AC, Aslan M, Ceylan MR, Olmez S, Karadas S, Akdeniz H. The syndrome of inappropriate secretion of antidiuretic hormone in patients with brucellosis. J Clin Lab Anal. 2015;29:366-9. doi: 10.1002/jcla.21780.

20. Aysha MH, Shayib MA. Syndrome of inappropriate secretion of antidiuretic hormone in brucellosis. J Infect. 1988;17:29-33. doi: 10.1016/s0163-4453(88)92260-8. 